\title{
Treatment of childhood acute lymphoblastic leukemia in Jakarta: Result of modified Indonesian National Protocol 94
}

\author{
Djajadiman Gatot, Endang Windiastuti
}

\begin{abstract}
Background Before 1990, the survival rates of childhood acute lymphoblastic leukemia (ALL) patients remained low. In 1994, the Hematology Oncology Working Group of the Indonesian Pediatric Association constructed a national protocol based on standard international protocol. As the outcome was still not promising, in 1998 the protocol was modified by introducing low dose MTX infusion for CNS prophylaxis.

Objective To analyze the survival of pediatric ALL patient treated with the modified protocol in Cipto Mangunkusumo Hospital, Jakarta.

Methods A prospective study was carried out to all newly diagnosed and relapsed children with ALL from January 1998 through December 2004. Patients were stratified into standard risk group (SRG) and high risk group (HRG). HRG met with one of these criteria: WBC $>50000 / i l$, the presence of CNS involvement, mediastinal mass, relapse, or $\mathrm{L}_{3}$ morphology. After completing induction therapy, all patients received low-dose MTX (LDMTX) infusion $\left(500 \mathrm{mg} / \mathrm{m}^{2}\right)$, especially for those aged less than 3 years. If the patient could not afford LDMTX, cranial irradiation (CRT) was given.

Results There were 309 patients, consisted of 190 SRG and 119 HRG patients. Male to female ratio was 1.8:1. Complete remission was achieved in $86.3 \%$ SRG patients compared with $63.8 \%$ in HRG patients $(\mathrm{P}<0.05)$. Event-free survival $(\mathrm{EFS})$ rate in $\mathrm{SRG}$ and $\mathrm{HRG}$ were $65.9 \%(95 \% \mathrm{Cl} 59.8 ; 71.9 \%)$ and $40.4 \%(95 \% \mathrm{Cl} 32.5 ; 48.4 \%)$, respectively. The overall survival (OS) rates in SRG was $81.2 \%(95 \% \mathrm{Cl} 76.3$; $86.2 \%)$ and in $\mathrm{HRG}$ was $56.0 \%(95 \% \mathrm{Cl} 47.8 ; 64.2 \%)$. The overall OS and EFS for both groups were $71.6 \%(95 \% \mathrm{Cl} 67.0 ; 76.2 \%)$ and $59.6 \%$ $(95 \% \mathrm{Cl} 54.5 ; 64.7 \%)$, respectively. Failure of therapy was mostly due to severe aplasia resulted in bleeding and severe infection. CNS relapse was rare in both groups, i.e. 3.1\% in SRG and $0.8 \%$ in HRG.

Conclusion Treatment of ALL using modified national protocol for SRG shows promising results. However, the outcome of HRG patients is still inferior to those reported elsewhere. The use of lowdose MTX infusion can replace the role of cranial irradiation as CNS prophylaxis measure. [Paediatr Indones 2006;46:179-184].
\end{abstract}

Keywords: acute lymphoblastic leukemia, childhood, survival,

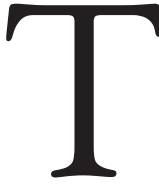

he outcome of children with acute lymphoblastic leukemia (ALL) has been improving dramatically during the last twenty years and approximately $80 \%$ of patients are cured. The treatment strategy for improving cure is made by applying multidrugs regimen as well as using high dose chemotherapy. Current strategy is to modify the intensity of therapy based on the acquired genetic characteristics of leukemia, using more intensive therapy for ALL with molecular markers, indicating a resistant leukemia. ${ }^{1}$

To improve the outcome of children with ALL, in 1994, The Working Group of The Indonesian Pediatric Hematology Oncology designed a national protocol based on standard ALL protocol with the aim that the treatment would be well tolerated, less severe toxicity, and lower cost. This is important because of unavailability of isolation ward, less optimal supportive care, and expensive cost of cytostatic drugs. The induction therapy was still quite toxic, as many

From the Department of Child Health, Medical School, University of Indonesia, Jakarta, Indonesia.

Reprint requests to: Djajadiman Gatot, MD, Hematology-Oncology Division, Department of Child Health, Medical School, University of Indonesia, Cipto Mangunkusumo Hospital, Jl. Salemba 6, Jakarta, Indonesia. Tel.62-21-31091170. Email: djagat_ps@yahoo.com 
of patients died from complications due to severe aplasia, in 1998 we again modified this protocol by reducing the course of L-asparaginase (from 9 to 6 courses) and using low-dose of methotrexate (MTX, 500 mg/ $\mathrm{m}^{2}$ ) by infusion for the central nervous system (CNS) prophylaxis, especially for those under 3 years of age in order to reduce late neurological sequelae.

In this study, we report the results of treatment of our pediatric patients with acute lymphoblastic leukemia using the modified national protocol. The protocol was the first protocol designed by The Hematology Oncology Working Group of The Indonesian Pediatric Hematology Oncology for the use nationwide.

\section{Methods}

All patients less than 18 years of age with ALL (newly diagnosed or relapsed) between January 1998 and December 2004, who were registered in the Division of Hematology Oncology, Department of Child Health, Cipto Mangunkusumo Hospital (CMH), Jakarta, were eligible for study. Infants less than 1 year of age were excluded in the analysis. Physical examination and complete blood count and morphology, bone marrow aspiration (BMA) were performed to all patients. The diagnosis of ALL was established when $30 \%$ or more lymphoblasts present in the bone marrow specimen and the type was defined using French-American-British (FAB) classification while immunophenotyping and cytogenetic study were not routinely performed due to its expensive cost. CNS leukemia was defined as the presence of at least 5 blasts $/ \mu \mathrm{l}$ in CSF.
At presentation, each patient was stratified into two different groups (standard risk and high risk). The high risk group (HRG) in our study met with one of these criteria: hyperleukocytosis (WBC >50 000/ $\mu$ l), CNS leukemia, mediastinal mass, $\mathrm{L}_{3}$ morphology, and relapse.

The regimen of protocols was illustrated in Figure 1. In brief, early induction therapy was consisted of dexamethasone (dexa), vincristine (VCR), daunorubicin (DNR), L-aspara-ginase (L-asp), and IT MTX. Remission induction therapy was the same for all patients, but CNS prophylaxis and intensification differed by risk category as could be seen in Figure 1. BMA examination was done at the end of remission induction therapy (week 6). When remission was achieved, then patients received CNS prophylactic treatment consisting of IT chemotherapy and either systemic MTX or cranial irradiation (CRT). For patients younger than 3 years of age, intravenous infusion of $500 \mathrm{mg} / \mathrm{m}^{2}$ MTX was given instead of CRT. Patients older than 3 years of age received either MTX infusion or 1800 cgy of CRT.

The standard risk group (SRG) received maintenance therapy at week 10 , while the HRG received it after the intensification therapy. The duration of therapy was 2 years. Complete remission (CR) was defined as the absence of blast cells in peripheral blood and CSF, bone marrow with $\geq 5 \%$ blasts, absolute neutrophil count $\geq 1000 / \mu \mathrm{l}$, platelets $\geq 100000 / \mu \mathrm{l}$, and no evidence of disease at any other site. Relapse was defined as the recurrence of lymphoblasts $(\geq 20 \%$ blasts cells in the bone marrow) or localized leukemic infiltration at any site after remission was achieved.

The Kaplan and Meier analysis was used to estimate survival rates. Overall survival (OS) was estimated

SRG

\begin{tabular}{|l|l|l|}
\hline & & \\
\hline Induction & CNS \\
Prophylaxis & Maintenance
\end{tabular}

HRG

\begin{tabular}{|l|l|l|l|}
\hline & & & \\
\hline Induction CNS Intensification CRT & Maintenance \\
Prophylaxis
\end{tabular}

\begin{tabular}{lllll}
\hline Week & 6 & 10 & 16 & 20
\end{tabular}

104

Figure 1. OUtLine of national PROTOCOL OF CHILDHOOd ALL 
using the duration from the start of treatment to the end of follow up time. Event-free survival (EFS), as the usual gold standard measure of clinical trial outcome, was estimated using the time from CR achievement to relapse, death, or lost to follow-up. Data were analyzed by SPSS for window (version released 13).

\section{Results}

A total number of patients were 309, consisted of 190 patients of SRG and 119 patients of HRG. Clinical and laboratory characteristics of the two groups of patients are listed in Table 1. The median follow-up was 19 months (1-74 months). Male to female ratio was 1.8:1 with median age 4.6 years. Hepatosplenomegaly was more frequent in the SRG. Laboratory findings showed that 118 out of 190 patients in the SRG (62.1\%) presented with $\mathrm{WBC}<10000 / \mu \mathrm{l}$, while in the HRG, $53.7 \%$ with WBC $>50000 / \mu$ l. None of the patients in the SRG had CNS leukemia at time of diagnosis compared with 11 cases in the HRG.

Table 2 summarized the findings of remission induction therapy and subsequent courses of therapy of the two groups. Complete remission was achieved in $86.3 \%$ in SRG patients, which was significantly better than $63.8 \%$ in $H R G$ patients $(\mathrm{P}<0.05)$. Failure of remission or death during induction therapy
Table 1. Clinical and laboratory characteristics

\begin{tabular}{|c|c|c|c|}
\hline Variable & Standard Risk & High Risk & Total \\
\hline \multicolumn{4}{|l|}{ Gender } \\
\hline Male & 122 & 75 & 197 \\
\hline Female & 68 & 44 & 112 \\
\hline \multicolumn{4}{|l|}{ Age (year) } \\
\hline 0 & 11 & 4 & $15^{*}$ \\
\hline $1-$ & 28 & 13 & 41 \\
\hline $2-$ & 139 & 77 & 216 \\
\hline$\geq 9$ & 23 & 29 & 52 \\
\hline \multicolumn{4}{|l|}{ Hepatomegaly } \\
\hline Yes & 145 & 101 & 246 \\
\hline No & 45 & 18 & 63 \\
\hline \multicolumn{4}{|l|}{ Splenomegaly } \\
\hline Yes & 130 & 98 & 228 \\
\hline No & 60 & 21 & 81 \\
\hline \multicolumn{4}{|l|}{ 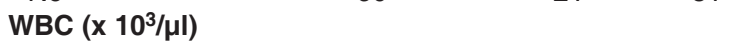 } \\
\hline$<10$ & 118 & 25 & 143 \\
\hline $10-50$ & 72 & 30 & 102 \\
\hline $50-100$ & 0 & 26 & 26 \\
\hline$>100$ & 0 & 38 & 38 \\
\hline CNS involvement & 0 & 11 & 11 \\
\hline Mediastinal mass & 0 & 3 & 3 \\
\hline \multicolumn{4}{|l|}{ FAB } \\
\hline L1 & 181 & 99 & 280 \\
\hline L2 & 9 & 1 & 10 \\
\hline L3 & 0 & 19 & 19 \\
\hline
\end{tabular}

* Not analyzed

was observed in $42 \mathrm{HRG}$ patients (35.2\%) and $26 \mathrm{SRG}$ patients (13.7\%). Causes of death were massive bleeding in 26 patients, septicemia in 23 patients, seizures in 12 patients, and other concomitant severe infections in 7 patients.

Table 2. Treatment Results

\begin{tabular}{|c|c|c|}
\hline Variable & SRG & HRG \\
\hline No of patient & 190 & 119 \\
\hline Complete remission (CR) & $164(86.3 \%)$ & $77(63.8 \%)$ \\
\hline \multicolumn{3}{|l|}{ Relapse } \\
\hline Total & $34(17.8 \%)$ & $22(18.5 \%)$ \\
\hline Bone marrow & 25 & 21 \\
\hline CNS & 3 & 1 \\
\hline $\mathrm{BM}+\mathrm{CNS}$ & 3 & 0 \\
\hline $\mathrm{BM}+$ testis & 1 & 0 \\
\hline Testis & 2 & 0 \\
\hline \multicolumn{3}{|l|}{ Death } \\
\hline During induction therapy & $26(13.7 \%)$ & $42(35.2 \%)$ \\
\hline During CR & 5 & 9 \\
\hline After relapse & 19 & 14 \\
\hline os & $81.2 \%$ (95\%Cl: 76.3; 86.2\%) & $56.0 \%$ (95\%Cl: $47.8 ; 64.2 \%)$ \\
\hline EFS & $65.9 \%$ (95\%Cl: 59.8; 71.9\%) & $40.4 \%(95 \% \mathrm{Cl}: 32.5 ; 48.4 \%)$ \\
\hline OS (SRG \& HRG) & \multirow{2}{*}{\multicolumn{2}{|c|}{$\begin{array}{l}71.6 \%(95 \% \mathrm{Cl}: 67.0 ; 76.2 \%) \\
59.6 \%(95 \% \mathrm{Cl}: 54.5 ; 64.7 \%)\end{array}$}} \\
\hline EFS (SRG + HRG) & & \\
\hline Lost to follow-up & 26 & 14 \\
\hline
\end{tabular}

HRG: high risk group; BM: bone marrow; CNS: central nervous system; OS: overall survival; EFS: event-free survival 
OS

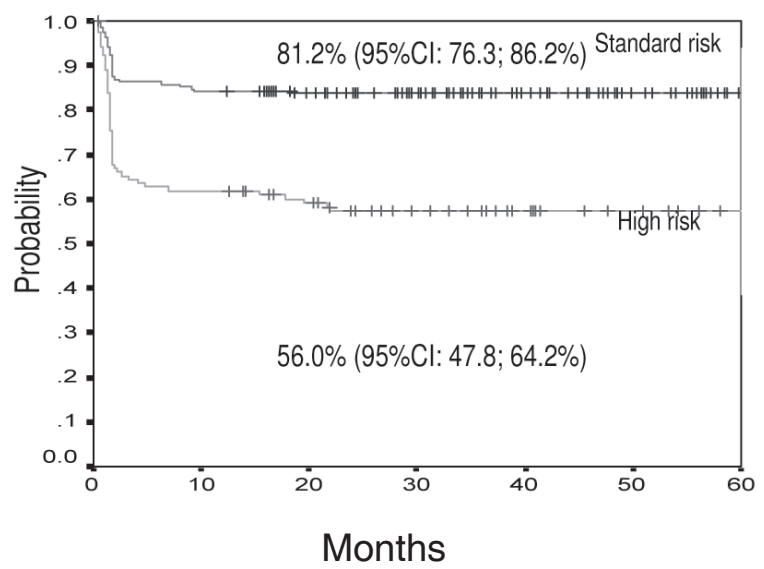

EFS

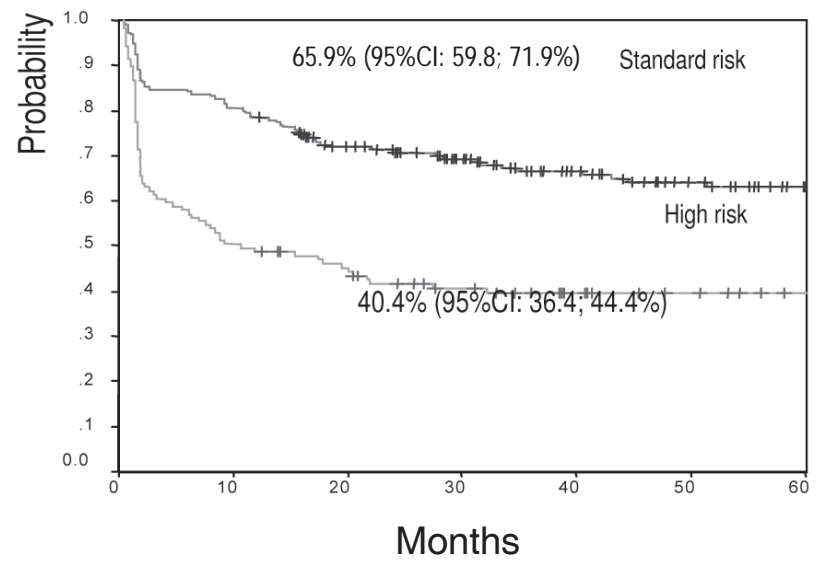

Figure 2A. Kaplan Meier estimates of overall survival and eVent-free survival according to stratification.

The relapse rate was $17.8 \%$ in SRG compared to $18.5 \%$ in HRG. The main site of relapse was bone marrow which were $13.2 \%$ and $18.5 \%$ for SRG and HRG, respectively. Total CNS relapse in SRG was observed in 6 patients, and 3 of them with bone marrow relapse, while in HRG was found only in 1 patient.

Event-free survival and survival curves were constructed by Kaplan and Meier method as seen in Figure $2 \mathrm{~A}$ for the SRG and HRG and in Figure 2B for all patients.

After a median observation time (19 months), the OS for SRG was $81.2 \%(95 \%$ CI $76.3 ; 86.2 \%)$ and for HRG was 56.0\% (95\%CI 47.8; 64.2\%\%). The estimate EFS for SRG was 65.9\% (95\%CI 59.8; 71.9\%) and for HRG was 40.4\% (95\%CI 36.4; 44.4\%) (Fig- ure 2A). The OS for all 309 patients was $71.6 \%$ (95\%CI 67.0; 76.2\%) and the estimate EFS was $59.6 \%$ (95\%CI 54.5; 64.7\%) (Figure 2B).

\section{Discussion}

This outcome data provided reasonably good result for SRG patients who were treated with less intensive chemotherapy for developing world like Indonesia with limited resources. ${ }^{2-5}$ Nevertheless, the survival of our patients is lower than that reported by other developing countries which adopt protocol from developed centres using intensive chemotherapy. ${ }^{6,7}$ Complete remission in SRG after
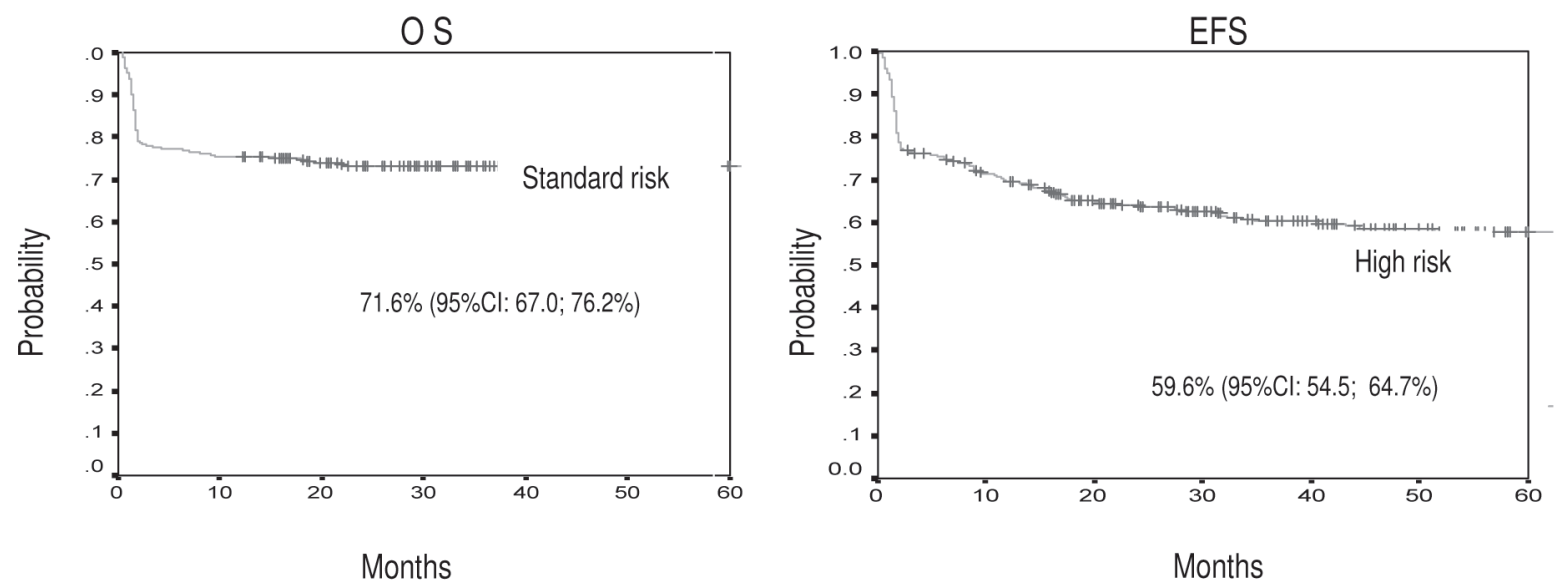

Figure 2B. Kaplan-Meier estimates of overall survival and eVEnt-Free survival in all Patients. 
the first remission induction in our study was $86.3 \%$. This is slightly lower than those treated with more intensive therapy which achieved CR higher than $90 \%$. $^{7-11}$

Figures $2 \mathrm{~A}$ and $2 \mathrm{~B}$ showed the overall survival (OS) and event-free survival (EFS) rate for the SRG. These results were not different from the outcome data of trial ALL-BFM 86. ${ }^{12}$ Decreased EFS was due to relapse and death during therapy as supportive care was not adequate. The result of therapy for HRG patients was still poor. The OS and EFS for this group was $56.0 \%$ and $40.4 \%$, respectively. This outcome was accepted because the induction and intensification therapy was less intensive and also there is no pharmacogenetics stratification of leukemic cell type. ${ }^{1}$ While using more intensive multidrugs therapy, the outcome of HRG was improved. 3,9-11 Overall survival for the two groups ( $\mathrm{SRG}+\mathrm{HRG}$ ) was 71.6 and EFS was $59.6 \%$. These results were very much better compared to results of our previous protocol when $\mathrm{L}$-asparaginase was not added in the induction therapy, ${ }^{13}$ but still lower than results from other countries as more intensive chemotherapy introduced. ${ }^{7-9,14}$ The use of $\mathrm{L}$-asparaginase was believe to increase remission rate and can maintain longterm remission, but in trial ALL-BFM 90, L-asparaginase did not improve outcome in the medium-risk group (MRG). ${ }^{8}$

One of the obstacles that reducing the outcome treatment was death mostly during induction therapy; several occurred during complete remission and again increase after relapse. The causes of deaths were uncontrolled bleeding, septicemia, convulsion, and other severe infections, which consists of 26, 23, 12 , and 7 patients, respectively. These were mainly due to severe aplasia. Bleeding, mostly as a consequence of very low platelet count $(<20000 / \mu \mathrm{l})$ sometimes can not be stopped promptly by platelet transfusion because of shortage of platelet concentrate in the blood bank. While septicemia or other infection were mainly due to nosocomial microorganism (i.e. Pseudomonas spp) which often difficult to overcome even with potent antibiotics. Convulsion or seizures with decrease consciousness were difficult to be assessed and may be caused by intracranial hemorrhage or by leukemic cells infiltration, especially when the onset was slow. Such condition were not seen in big treatment centers where the incidence of death was very low with proper facilities of hospitalization as well as adequate supportive care. ${ }^{8-10}$

Central nervous system (CNS) involvement was found only in the HRG at time of diagnosis (11 out of 75 patients) and was treated by IT MTX + ARA$\mathrm{C}+$ Dexa twice weekly until blast cells cleared from the CSF. The cranial irradiation was given only to those above 3 years of age or allergic to or can not afford MTX. In the SRG, 71 patients received cranial irradiation and 93 patients receive LDMTX for CNS prophylaxis. Unexpectedly, CNS relapses were observed more frequent in patients received CRT (5) than patients with LDMTX (1). This result can not be explained clearly. Interestingly, in the HRG, in which 11 patients had CNS involvement at time of diagnosis, only 1 patient developed CNS relapse; this was probably because all patients in SRG received both LDMTX infusion with IT chemotherapy and CRT for CNS prophylaxis.

In the ALL-BFM-86 study, it was reported that systemic medium-dose MTX and intrathecal chemotherapy can safely replace cranial irradiation in childhood ALL patients with low leukemic cell mass at diagnosis. ${ }^{15}$ This data outcome is comparable to our result even with LDMTX.

In conclusion, the result of modified National Protocol for SRG patient improved. On the other hand, the outcome of HRG patient was still inferior to those reported elsewhere. The use of low dose MTX infusion can replace the role of cranial irradiation as CNS prophylaxis measure.

\section{Acknowledgments}

We thank Ferry Damardjati, MD and Rosary, MD for their assistance in statistical analysis.

\section{References}

1. Rocha JCC, Cheng C, Liu W, Kishi S, Das S, Cook $\mathrm{EH}$, et al. Pharmacogenetics of outcome in children with acute lymphoblastic leukemia. Blood 2005;105:4752-8.

2. Shing MMK, Li CK, Chik KW, Lam TK, Lai HDH, Ling $\mathrm{Ng} \mathrm{MH}$, et al. Outcomes and prognostic factors 


\section{Paediatrica Indonesiana}

of chinese children with acute lymphoblastic leukemia in Hongkong: Preliminary results. Med Pediatr Oncol 1999;32:117-123.

3. Ribeiro RC, Pui CH. Saving the children-improving childhood cancer treatment in developing countries. N Engl J Med 2005;352:2158-60.

4. Howard SC, Pedrosa M, Lins M, Pedrosa A, Pui CH, Ribeiro RC, et al. Establishment of a pediatric oncology program and outcomes of childhood acute lymphoblastic leukemia in a resource-poor area. JAMA 2004;291:2471-5.

5. Laosombat V, Wongchanchailert M, Sattayasevana B, Wiriyasateinkul A, Watana-Arepornchai S. The treatment of children with acute lymphoblastic leukemia in Thailand. Med Pediatr Oncol 2002;38:266-8.

6. Campbell M, Salgado C, Quintana J, Becker A, Vargas L, Cabrera ME, et al. Improved outcome for acute lymphoblastic leukemia in children of a developing country: Results of the Chilean National Trial PINDA 87. Med Pediatr Oncol 1999;33:88-94.

7. LeClerc JM, Billett AL, Gelber RD, Dalton V, Tarbell N, Lipton JM, et al. Treatment of childhood acute lymphoblastic leukemia: Results of Dana-Farber ALL consortium protocol 87-01. J Clin Oncol 2001;20:237-46.

8. Schrappe M, Reiter A, Ludwig WD, Harbott J, Zimmermann M, Hiddemann W, et al. Improved outcome in childhood acute lymphoblastic leukemia despite reduced use of anthracyclines and cranial radiotherapy: Results of trial ALL-BFM 90. Blood 2000;95:3310-22.

9. Kamps WA, Bokkerink JPM, Hahlen J, Riehm H, Gadner H, Schrappe M, et al. Intensive treatment of children with acute lymphoblastic leukemia according to ALL-BFM-86 without cranial radio- therapy: Results of Dutch childhood leukemia study group protocol ALL-7 (1988-1991). Blood 1999;94: 1226-36.

10. Arico M, Valsecchi MG, Conter V, Rizzari C, Pession A, Messina C, et al. Improved outcome in high-risk childhood acute lymphoblastic leukemia defined by prednisone-poor response treated with double Berlin-Frankfurt-Muenster protocol II. Blood 2002;100:420-6.

11. Ishii E, Eguchi H, Matsuzaki A, Koga H, Yanai F, Kuroda $\mathrm{H}$, et al. Outcome of acute lymphoblastic leukemia in children with AL90 regimen: Impact of response to treatment and sex difference on prognostic factors. Med Pediatr Oncol 2001;37:10-9.

12. Reiter A, Schrappe M, Ludwig WD, Hiddemann W, Sauter S, Henze G, et al.Chemotherapy in 998 unselected childhood acute lymphoblastic leukemia patients: Results and conclusions of the multicenter trial ALL-BFM 86. Blood 1994;84:3122-33.

13. Abdulsalam M, Gatot D. The development of treatment of childhood acute lymphoblastic leukemia in Jakarta. Presented in the ESO-SIOP Pediatric Oncology Course. Surabaya, Indonesia 2003.

14. Freeman AI, Boyett JM, Glicksman AS, Brecher ML, Leventhal BG, Sinks LF, et al. Intermediate-dose methotrexate versus cranial irradiation in childhood acute lymphoblastic leukemia: A ten-year follow- up. Med Pediatr Oncol 1997;28:98-107.

15. Riehm H, Gadner H, Henze G, Kornhuber B, Lampert F, Niethammer D, et al. Results and significance of six randomized trials in four consecutive ALL-BFM studies. Haematol Blood Transfus 1990;33:439-50. 